\title{
Interdisziplinarität auf dem Prüfstand
}

nterdisziplinä res Arbeiten" hat sich zu einem politisch korrekten Modebegriff entwickelt.
Ihm haftet zur Zeit etwas "allgemein Gutes" an, so wie etwa dem "Klimaschutz" oder
dem "Nachhaltigkeitsprinzip". Mit der Popularität solcher Begriffe wird allerdings auch
deren Definition breiter; vielfach um den Preis abnehmender Aussagekraft. Dies führt dazu,
daß Kritiker heute interdisziplinä res Arbeiten oft als Deckmantel für oberflächliche Wissenschaft betrachten.

Für den jüngsten Jahreskongreß der Schweizerischen Akademie der Naturwissenschaften (SANW) in Freiburg im Uechtland hat die Freiburger Naturforschende Gesellschaft als Organisatorin den interdisziplinä ren Dialog auf den Prüfstand gestellt. Die SANW vereint gegenwärtig 44 Fachgesellschaften, 22 Kommissionen, 29 regionale und kantonale Gesellschaften sowie 8 thematische Plattformen und zählt rund 30000 Mitglieder. Als Organisation der Naturwissenschaften in der Schweiz ist sie den nationalen Aufgaben und Interessen der Naturwissenschaften verpflichtet, die oftmals disziplinenü bergreifenden Charakter haben. Das Zusammentreffen am Jahreskongreß findet jeweils unter einem gemeinsamen Thema statt. Es wurde in diesem Jahr so gestaltet, daß die Fachvorträ ge einem fachlich gemischten Publikum präsentiert wurden, um anschließend einen interdisziplinä ren Dialog zu führen. Das Organisationskomitee hat sich für das gesellschaftsrel evante Thema "Stürme überall" entschieden, denn Stürme - im konkreten wie im übertragenen Sinne - passieren sozusagen in allen Gebieten der Naturwissenschaften, aber auch in Gesellschaft, Wirtschaft und Kultur, und zwar nicht nur in jüngster Zeit. Der Sturm auf die Bastille am 14. Juli 1789, die Maschinenstürmer zu Beginn des 19. Jahrhunderts in England und auf dem Kontinent sowie schon die Bilderstürmer zur Zeit der Reformation im 16. Jahrhundert sind wichtige Ereignisse in der europäischen Geschichte.

Die Motive für die Wahl von Thema und Form des Kongresses waren innerwissenschaftlicher Natur. Es ging um den Nutzen des interdisziplinä ren Dialogs für das Verständnis der komplexen Phänomene bei stürmisch ablaufenden Prozessen. Wesentliche Merkmale der Interdisziplinarität sind es, Konzepte und Methoden verschiedener Disziplinen so zu kombinieren beziehungsweise auf andere Gebiete zu übertragen, damit die Erkenntnis in einem Problembereich zunimmt. Die Frage, wie die Gesellschaft mit Stürmen und ihren Folgen besser umgehen kann, stand nicht im Vordergrund. Sie hätte Transdisziplinaritä $t$ erfordert, was bedeutet, daß Problembereiche interdisziplinä $r$ und unter Einbezug der Bedürfnisse und Praktiken relevanter gesellschaftlicher Gruppen untersucht werden, um es der Gesellschaft zu ermöglichen, Entwicklungen zukünftig gezielt und besser zu gestalten.

\begin{tabular}{|c|c|}
\hline \\
\hline \multicolumn{2}{|l|}{$\begin{array}{l}\text { Glossar } \\
\text { Disziplinen }\end{array}$} \\
\hline Multidisziplinarität & $\begin{array}{l}\text { Ergebnisse verschiedener Disziplinen zu einem gemeinsamen } \\
\text { Forschungsthema stehen beziehungslos nebeneinander } \\
\text { (zum Beispiel: Ringvorlesungen). }\end{array}$ \\
\hline Interdisziplinarität & $\begin{array}{l}\text { Kombination und Übertragung von Konzepten und Methoden } \\
\text { verschiedener Disziplinen für Innovation in der Forschung } \\
\text { (zum Beispiel: Molekularbiologie). }\end{array}$ \\
\hline Transdisziplinarität & $\begin{array}{l}\text { Problembereiche werden interdisziplinär und unter Einbezug } \\
\text { der verschiedenen Bedürfnisse relevanter gesellschaftlicher } \\
\text { Gruppen untersucht. Die Erkenntnisse ermöglichen der } \\
\text { Gesellschaft, Entwicklungen gezielt und besser zu gestalten. }\end{array}$ \\
\hline rmale D & $\begin{array}{l}\text { Fachgebiete wie Statistik oder Modellierung, deren Verfahren } \\
\text { auf alle empirischen Problembereiche anwendbar sind. }\end{array}$ \\
\hline
\end{tabular}

Am Kongre 3 wurde aufgezeigt, welche Assoziationen nun Forschende zu einem Begriff wie "Sturm" haben und wie sie stürmisch ablaufende Prozesse in diversen Disziplinen beschreiben und strukturieren. Wir stellten uns die Kernfragen: Sind die für ein Gebiet identifizierten Entwicklungen und Mechanismen auf andere Disziplinen übertragbar? Können wir daraus voneinander lernen? Stellen sich "Aha-Erlebnisse" ein, oder fühlen wir uns eher in die Irre geführt, wenn wir die Konzepte von Stürmen in durchaus verschiedenen Bereichen miteinander vergleichen?

Fachvorträge zu ausgewählten Sturmthemen bildeten die Grundlage für drei Workshops und eine Podiumsdiskussion. Hierbei wurde erörtert, für welche Probleme in der Forschung Interdisziplinaritä $t$ notwendig ist, aber auch wo ihre Grenzen sind. Mit einem künstlerischen Begleitprogramm wurde der Horizont noch weiter geöffnet. Das Organisationsteam versprach sich von diesem Experiment Impulse für die Forschungskultur generell sowie einen Gewinn für die fachspezifische Forschung: Mehrwert durch Interdisziplinarität als Erkenntnis- und Erlebniswert.

Nicht unerwartet traten die Unterschiede der Fragestellungen und des methodischen Vorgehens zwischen Naturwissenschaften einerseits und Sozial- und Geisteswissenschaften anderseits deutlich zutage. Naturwissenschaftler beschreiben Stürme empirisch und sind gewohnt, zu strukturieren und mathematisch zu modellieren. Sie wollen Prognosen über das Entstehen, den Verlauf und die Folgen von Stürmen stellen. Für Sozial- und Geisteswissenschaftler hingegen steht bei Stürmen in der individuellen Entwicklung wie auch in Wirtschaft und Gesellschaft der Mensch im Zentrum. Die Medien spielen dabei eine große Rolle, indem sie darauf Einfluß 
nehmen, daß Ereignisse und die durch sie ausgelösten Gefühle als Stürme wahrgenommen und in Handlungen umgesetzt werden. Stürme in der individuellen oder gesellschaftlichen Entwicklung werden oft als Krise des Ordnungsund Orientierungsgefü ges empfunden, wobei nach Sinn und Verantwortung gefragt wird. Die Diskussionen führten zu Fragen wie: Läßt sich die Komplexität stürmischer Prozesse in der Natur auch als Störung grundlegender Gleichgewichtsbeziehungen beschreiben? Wie beeinflußt das menschliche Empfinden, das beim wertenden Begriff des Sturms nicht wegzudenken ist, die naturwissenschaftliche Untersuchung von Stürmen? Oder anders gefragt: Untersuchen die Naturwissenschaften tatsächlich Stürme?

Doch bilden Sie sich Ihr eigenes Urteil: Die in dieser GAIA-Sondernummer zusammengestellten Beiträge sind Kurzfassungen der Kongreßvorträge. Einführend wird die Bedeutung von Metaphern in der naturwissenschaftlichen Forschung erörtert, insbesondere wie Metaphern als Brücken zwischen Fachgebieten funktionieren und wie sie auch forschungsleitend wirken können. Die naturwissenschaftlichen Fachbeiträge von Nachwuchsforschende n über Stürme in der Natur sowie über Stürme im Universum und im Laboratorium zeigen beispielhaft, daß Stürme in vielen Bereichen und Dimensionen vorkommen und nicht einfach auf meteorologische Ereignisse reduzierbar sind. In den Syntheseartikeln sind durch ausgewiesene Fachexperten zentrale Gemeinsamkeiten und Unterschiede der naturwissenschaftlichen Beiträge herausgearbeitet. Ein weiterer Synthesebeitrag kommentiert die vier Vorträge über Stürme in der persönlichen Entwicklung, der Gesellschaft, der Kunst und Kultur sowie in der Politik.

Auf dem Prüfstand, der in einer stürmischen Auseinandersetzung mit "Stürmen überall" bestand, sind nicht zuletzt auch Sinn und Grenzen des interdisziplinä ren Dialogs an einem Akademie-Kongreß deutlich geworden. Das Thema "Stürme überall" hat hervorragende Nachwuchsforschende angesprochen, aber auch das Interesse von Arrivierten und von Persönlichkeiten in der Gesellschaft geweckt. Im interdisziplinä ren Dialog konnten die großen Linien abgesteckt werden, die als Einsichten befruchten. Ein konkreter Nutzen in der Forschung verlangt jedoch "Knochenarbeit", die nicht am Kongreß stattfindet. Die fruchtbare Zusammenarbeit mit anderen Fachgebieten im Forschungsalltag setzt ein gewisses Verständnis für diese Gebiete voraus. Der fortwährende Zwang zur Spezialisierung und Zeitdruck sind aber eine Hürde. Wenn sich das Bekenntnis zu Interdisziplinaritä t auf die Forschungspraxis auswirken soll, dann bedarf es dafür nicht in erster Linie einer von oben verordneten Aufforderung zur fachübergreifenden Zusammenarbeit. Nachwuchswissenschaftler wie auch Fachexperten und Forschungsleiter waren sich einig, daß einer gezielten Schulung von Kompetenzen bereits während des Studiums vermehrte Aufmerksamkeit geschenkt werden sollte. Damit sich das Bekenntnis zu Interdisziplinaritä $t$ auf die Forschungspraxis auswirkt, müssen in der akademischen Laufbahn auch institutionelle Anreize geboten und spezifische interdisziplinäre Forschungsprogramme lanciert werden.

\section{Heinz Müller-Schärer ${ }^{1)}$, Reinhard Lässig ${ }^{2}$} und Gertrude Hirsch Hadorn ${ }^{3}$

1) Präsident der Freiburger Naturforschenden Gesellschaft (2000-2002); Département de Biologie/Ecologie \& Evolution, Université de Fribourg, CH-1700 Fribourg; heinz.mueller@unifr.ch.

2) Forschungsbereich Wald, Eidgenössische Forschungsanstalt für Wald, Schnee und Landschaft, CH-8903 Birmensdorf; laessig@wsl.ch.

3) Vizepräsidentin der Schweizerischen Akademie der Naturwissenschaften Departement Umweltnaturwissen schaften, ETH Zürich, CH-8092 Zürich; hirsch@umnw.ethz.ch.

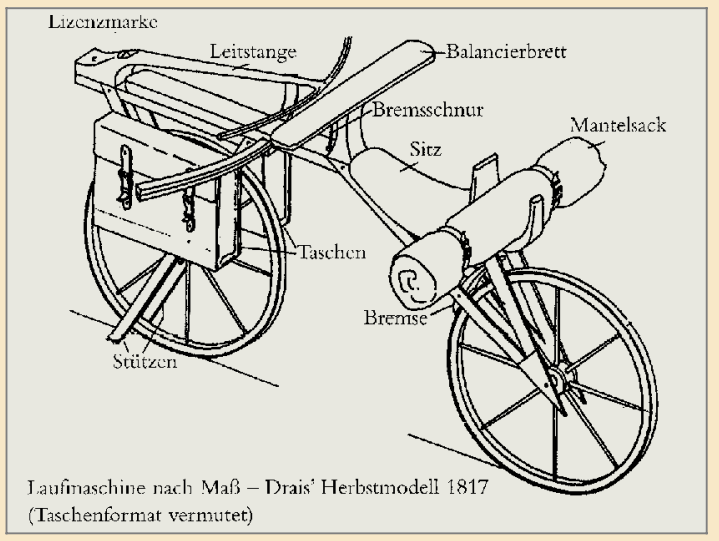

Eine Fahrradwissenschaft, wenn es sie gäbe, hätte wie jede ordentliche Wissenschaft einen klar definierten Gegenstand und dazu forschungsdienliche Modelle. Wie steht es in dieser Hinsicht um die Umweltwissenschaft?

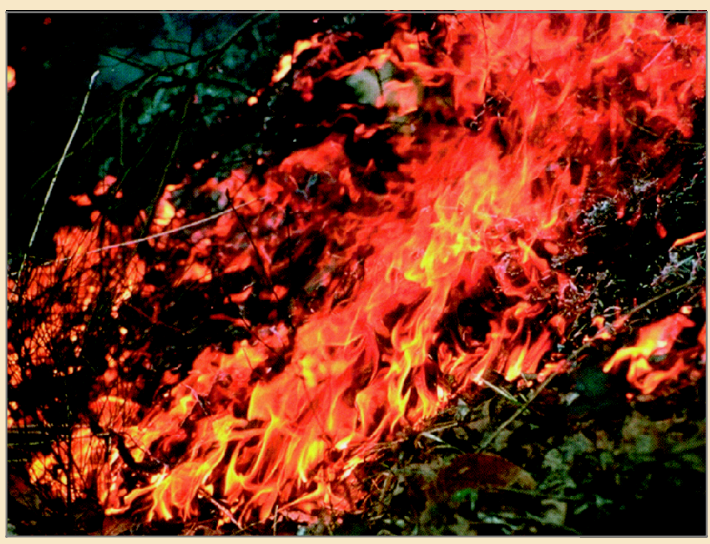

Im Zentrum des Jahreskongresses der Schweizerischen Akademie der Naturwissenschaften (SANW) stand eine Metapher die Metapher vom Sturm - , um einen interdisziplinären Dialog zu katalysieren. Was verbindet Stürme in der Natur ...
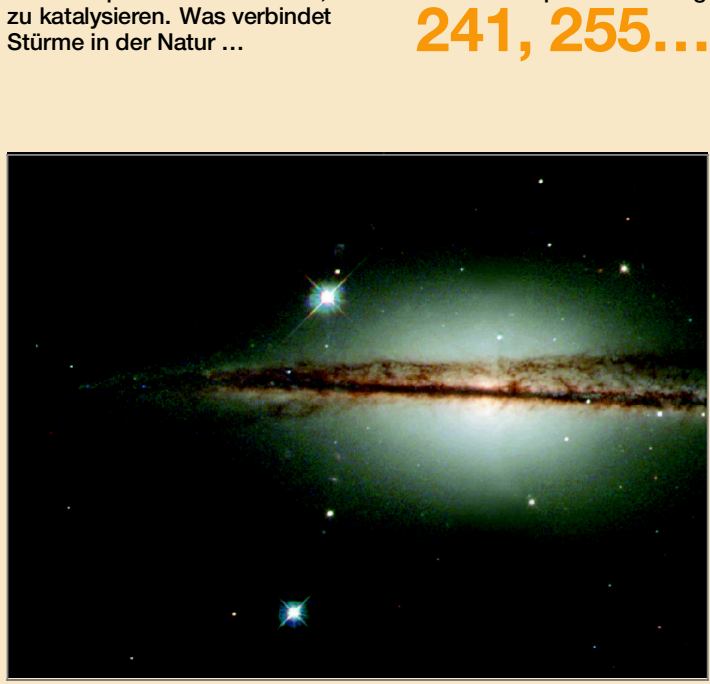

... mit Stürmen im Universum, wie sind Stürme nachzuvollziehen, wie ihre Folgen für den Menschen zu bewerten und zu mindern, wie ist ihr Auftreten vorherzusagen - und welche Analogien bestehen zu Stürmen in der Gesellschaft? 